\title{
Parámetros de calidad durante la cosecha de durazno (Prunus persica L. Batsch Cv. "Rubidoux")
}

\section{Quality parameters during the harvest of peach (Prunus persica L. Batsch cv. "Rubidoux")}

\author{
Parâmetros de qualidade durante a colheita de pêssego \\ (Prunus persica L. Batsch cv. "Rubidoux")
}

\author{
Lisney Alessandra Bastidas Parrado', Gloria Acened Puentes Montañez², Angela Lemus Cerón ${ }^{3}$ \\ 1,2, 3 Escuela Administración de Empresas Agropecuarias. Facultad Seccional Duitama. Universidad \\ Pedagógica y Tecnológica de Colombia. Carrera 18 Calle 22, Duitama. Boyacá. Colombia. \\ Grupo de Investigación CERES. Escuela Administración de Empresas Agropecuarias UPTC- Duita- \\ ma. Facultad Seccional Duitama. Carrera 18 Calle 22, Duitama, Boyacá, Colombia.

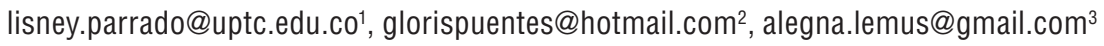

\begin{abstract}
Resumen
El durazno es una fruta de importancia agrícola como parte de los cultivos caducifolios producidos en Colombia; su calidad y aceptación comercial, así como el momento de la cosecha, están definidos en función de sus características físicoquímicas. Esta investigación tuvo como propósito caracterizar algunos parámetros de calidad de durazno variedad Rubidoux durante el período de cosecha, a partir de la selección hecha por operarios en campo, de acuerdo al color de la piel como indicador de madurez. Durante seis semanas de la cosecha se evaluaron muestras de frutos, considerando aspectos como peso fresco, contenido de sólidos solubles (Brix), categoría comercial de acuerdo al diámetro ecuatorial y color del fruto. Se registró un peso fresco promedio de 130,7 g por fruto, con un contenido de sólidos solubles promedio de 11,8 ${ }^{\circ}$ Brix, parámetro que incrementó progresivamente de la semana uno a la cinco. La clasificación visual por el color de la piel permitió definir siete categorías, tres predominaron durante la cosecha (pintón, pintón-maduro y maduro), estos se correspondieron con el contenido de
\end{abstract}

sólidos solubles; es así como el análisis de varianza mostró diferencias estadísticas entre Brix y el color de piel. Durante el período evaluado, se cosecharon con mayor frecuencia duraznos pintones y frutos con diámetro entre $60 \mathrm{~mm}$ a $70 \mathrm{~mm}$.

Palabras clave: color de piel, contenido de sólidos solubles, frutales caducifolios, índice de madurez.

\section{Abstract}

Peach is a fruit of agricultural importance as part of the deciduous crops produced in Colombia; its quality and commercial acceptance, as well as the time of harvest, are defined according to their physic-chemical characteristics. This research aimed to characterize some parameters of quality of Rubidoux peach during the harvest period, based on the selection made by operators in the field, according to the skin color as an indicator of maturity. For six weeks of the harvest samples of fruit were evaluated, considering aspects such as fresh weight, soluble solids content ( ${ }^{\circ}$ Brix), commercial-grade according to the equatorial 
diameter and skin color. An average of $130.7 \mathrm{~g}$ per fruit fresh weight was recorded, with a content of soluble solids of $11.8^{\circ}$ Brix, parameter that increased progressively from week one to the five. The visual classification by skin color allowed to define six categories, three of them predominated during the harvest (semi-ripe, semi-ripe-mature and mature), these corresponded with the content of soluble solids; this way, the analysis of variance

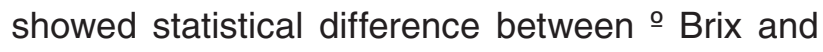
skin color. During the evaluated period, semi-ripe peaches between $60 \mathrm{~mm}$ to $70 \mathrm{~mm}$ were harvested more often.

Key-words: skin color, soluble solids content, deciduous fruit, maturity index.

\section{Resumo:}

O pêssego é uma fruta de importância agrícola que conforma as culturas de caducifólios produzidos na Colômbia; sua qualidade e aceitação comercial, bem como o momento da colheita, estão definidos em função das suas características físico-químicas. Esta pesquisa teve como objetivo caracterizar alguns parâmetros de qualidade de pêssego varieda- de Rubidoux durante o período da colheita, a partir da seleção feita por operários em campo, de acordo como a cor da casca como indicador de amadurecimento. Durante seis semanas de colheita, se avaliaram amostras de frutos, considerando aspectos como peso fresco, conteúdo de sólidos solúveis ( ${ }^{\circ}$ Brix), categoria comercial de acordo ao diâmetro equatorial e cor do fruto. Registrou-se um peso médio de $130.7 \mathrm{~g}$ por fruto, com um conteúdo de sólidos solúveis, em média, de $11.8^{\circ}$ Brix, parâmetro que acrescentou progressivamente da semana um a cinco. A classificação visual pela cor da casca permitiu definir sete categorias, três predominaram durante a colheita (pintado, pintado maduro e maduro), que corresponderam com o conteúdo de sólidos solúveis; é assim como a analise de variância mostrou diferenças significativas entre ${ }^{\circ}$ Brix e cor da casca. Durante o período avaliado, se colheram com maior freqüência pêssegos pintados e frutos com diâmetro entre $60 \mathrm{~mm}$ e $70 \mathrm{~mm}$.

Palavras-chave: cor de casca, conteúdo de sólidos solúveis, frutais caducifólios, índice de amadurecimento.

\section{Introducción}

La producción de caducifolios es una actividad agrícola rentable, genera oportunidades de negocio para los agricultores frente a otras alternativas de inversión de las regiones productoras, en especial el cultivo de durazno es visto como fuente económica atractiva para estos agricultores (Puentes et al., 2008), de acuerdo a la Sociedad Colombiana de Ciencias Hortícolas en Colombia $(\mathrm{SCCH})$ la especie predominante cultivada es el durazno (SCCH, 2012 citado por Ávila et al., 2013) y el departamento de Boyacá concentra un $60,5 \%$ la producción nacional (MADR, 2012). Las varie- dades predominantes de durazno en el departamento de Boyacá son Dorado, Diamante, Rey Negro y Rubidoux (Miranda \& Carranza, 2013), materiales que fueron establecidos en la Granja Tunguavita de la Universidad Pedagógica y Tecnológica de Colombia y dentro de la cual se cuenta con una huerta comercial de 4 ha con el material Rubidoux, el cual sirve como referente para otros productores de la región.

Una de las labores determinantes de este sistema de producción corresponde al momento de la cose- 
cha, el cual que está relacionado con su madurez, entendido como un estado donde el producto ha alcanzado un desarrollo suficiente, para que luego de la cosecha y el manejo poscosecha, su calidad sea la mínima aceptable para el consumidor final (Reid, 2002), generando así un grado de satisfacción al consumir el producto (Crisosto \& Valero, 2008). Existen diferentes parámetros que han sido utilizados para determinar el grado de madurez de frutales considerados como índices de madurez (Dagar et al., 2011; Daza et al., 2008; Ferrer et al., 2005) estos índices son importantes por las regulaciones comerciales que establecen el grado de madurez mínimo o máximo aceptado, se utilizan también como estrategia para la comercialización de productos y su conocimiento permite planear la cosecha del producto (Crisosto \& Valero, 2008).

Entre los parámetros más usados para definir la calidad de frutales están los físicos como el tamaño, la forma, peso, color y los químicos como acidez, contenido de azúcar, así como algunos compuestos volátiles que determinan su aroma (Byrne et al., 1991; Jha et al., 2010) Existen diversos estudios enfocados en determinar este tipo de parámetros de madurez en nectarinas y duraznos, partiendo de técnicas destructivas y no destructivas cuyo fin último es determinar el momento de la cosecha apropiado para que el producto recolectado sea aceptado comercialmente (Byrne et al., 1991; Pérez-Marín et al., 2009; Tijskens et al., 2007)using near-infrared (NIR dado que el fruto es altamente perecedero (Crisosto et al., 2009). Para este sistema de cultivo, en Colombia tradicionalmente se determina el índice de madurez de manera visual, identificando principalmente cambios de color en la piel del durazno, índice que en algunos cultivares es enmascarado porque se genera un cambio de color del exocarpio relacionado con la presencia de luz (Crisosto \& Costa, 2008; Kays, 1999).
Conociendo la importancia de este cultivo y los métodos de selección para la cosecha, esta investigación se propuso caracterizar algunos parámetros de calidad del durazno Rubidoux tales como sólidos solubles totales, y la frecuencia por categoría comercial, de acuerdo al diámetro ecuatorial y peso de los frutos, así como color de la corteza con el fin de tener información preliminar de esta variedad.

\section{Materiales y métodos}

El estudio se adelantó en una huerta de durazno Rubidoux de 10 años, durante la cosecha, entre los meses de marzo a mayo de 2013 en la Granja Experimental Tunguavita de la Universidad Pedagógica y Tecnológica de Colombia (UPTC) ubicada en la vereda El Salitre del municipio de Paipa, Boyacá, Colombia, a 5\%44' de latitud Norte y 73066'de longitud Oeste, a una altitud de 2.470 msnm; durante el período evaluado se presentaron precipitaciones totales de $295,4 \mathrm{~mm}$ y 244,9 horas de brillo solar (IDEAM, 2013).

La muestra correspondió a seis canastas de duraznos cosechados sin seleccionar y escogidas al azar, a razón de una por pase de cosecha, se entiende por pase cada etapa o semana donde se hizo la recolección del total de los árboles de la huerta (Usenik et al., 2008), material que fue transportado al Laboratorio de Poscosecha de la Escuela de Administración de Empresas AgropecuariasUptc, para ser analizado de manera inmediata.

Inicialmente se estableció una carta de color para la variedad Rubidoux de acuerdo a la proporción visual de color de piel y su intensidad sobre la zona ecuatorial (Casierra-Posada \& Aguilar-Avendaño, 2008). Se determinaron las categorías para comercialización en fresco respecto al diámetro ecuatorial de los frutos con un calibrador "pie de 
rey" digital MITUTOYO CD- 6" C5, con base en la selección hecha por el productor en campo para su distribución. En el laboratorio se clasificaron los frutos de acuerdo a las categorías comerciales previamente definidas y se pesaron en una balanza electrónica de piso modelo TCS - AE300 , a partir de los cuales y teniendo en cuenta la cantidad de frutos se estimó el peso fresco promedio de fruto y la frecuencia (\%) por categoría (Weber et al., 2003). Cada categoría se clasificó de acuerdo a la carta de colores separando los pintones, de pintones-maduros y maduros, haciendo un conteo de acuerdo a este parámetro. Se separaron tres frutos al azar de cada una de las categorías relacionadas con el color de la corteza, por cada uno de los seis pases, para los cuales se determinó ${ }^{\circ}$ Brix con un refractómetro BRIXCO FG113 (rango 0-32Brix). Esta información se analizó mediante el programa estadístico $R$, versión 2.12.2, con el cual se realizó análisis de varianza clásico, previa comparación de la normalidad de datos y homogeneidad de varianzas, seguido del test de comparación de medias de Tukey con un nivel de significancia $p \leq 0,05$.

\section{Resultados}

Una vez que la comercialización en fresco del durazno fue determinada por la apariencia y el color, se realizó una clasificación visual colorimétrica para el durazno Rubidoux. De esta manera se obtuvieron seis categorías para reflejar los estados de madurez desde el fruto denominado verde, de coloración intensa, hasta el sobremaduro con tonalidades amarillas-naranjas en más del $80 \%$ del fruto (Tabla 1).
Tabla 1. Grado de madurez de acuerdo al color para durazno Rubidoux en la Granja Experimental Tunguavita, Paipa, Boyacá

\begin{tabular}{lcc}
\hline \multirow{2}{*}{ Grado de madurez } & \multicolumn{2}{c}{ Color \% } \\
\cline { 2 - 3 } & Verde & Rojo \\
\hline Verde & 100 & 0 \\
\hline Verde pintón & 80 & 20 \\
\hline Pintón & 60 & 40 \\
\hline Pintón maduro & 40 & 60 \\
\hline Maduro & 20 & 80 \\
\hline Sobremaduro & 0 & 100 \\
\hline
\end{tabular}

Durante esta investigación se clasificaron los frutos recolectados en las categorías extra, primera, segunda y corriente, acorde a la clasificación hecha previamente por el agricultor dueño de la finca. Es así como en laboratorio se correspondió cada categoría con un rango de medida relacionado con el diámetro ecuatorial de los frutos, de manera que la categoría extra se correspondió con frutos cuyo diámetro fue mayor a $70 \mathrm{~mm}$, la categoría primera fue de $60-70 \mathrm{~mm}$, la segunda de $50-60 \mathrm{~mm}$ y la corriente menor de $50 \mathrm{~mm}$ de diámetro.

La muestra evaluada correspondió a 801 frutos provenientes de seis pases durante la cosecha. Se obtuvo así que la mayor frecuencia de frutos se correspondió con la categoría primera con un 51,8\%, seguido de la categoría segunda con $31,5 \%$, extra con $13,5 \%$ y corriente con $3,2 \%$ (Figura 1 ). 


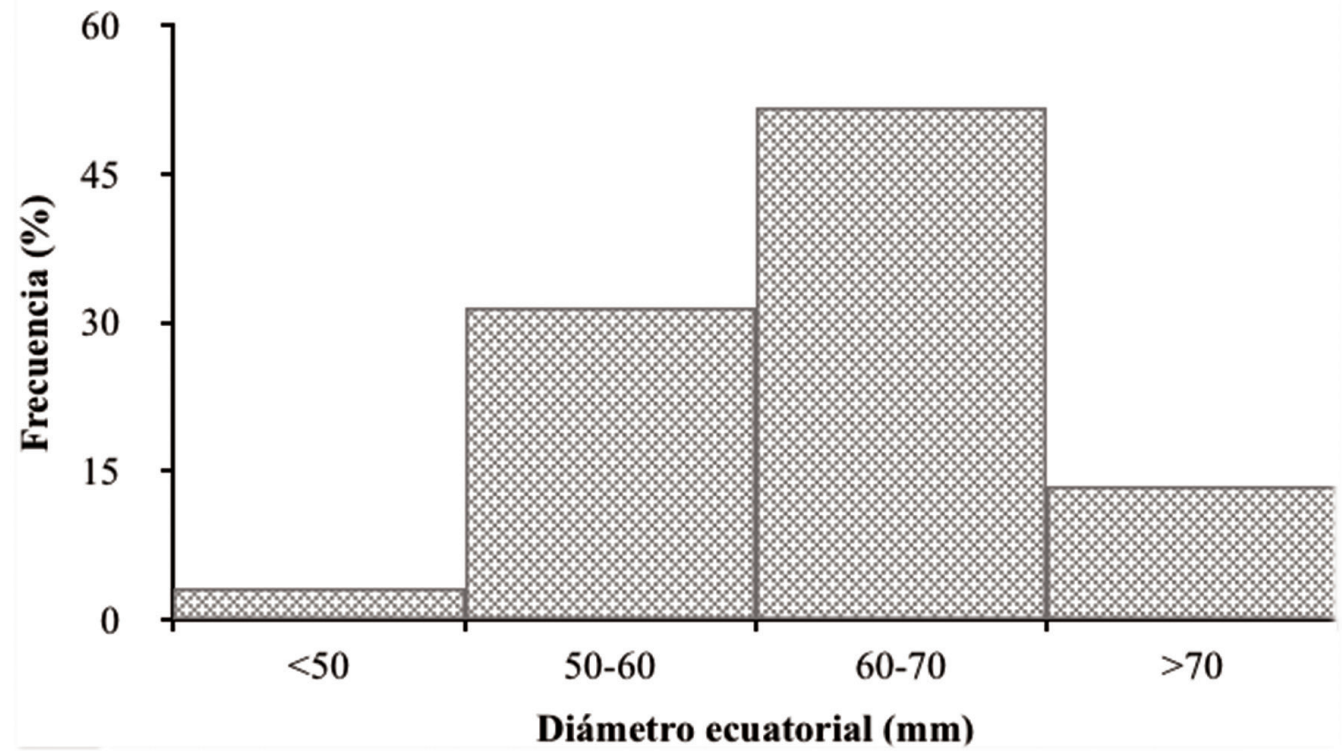

Figura 1. Distribución de las frecuencias de acuerdo al diámetro ecuatorial de frutos de duraznos Rubidoux cosechados en la Granja Experimental Tunguavita, Paipa, Boyacá.

Así mismo, se determinó el peso fresco promedio por fruto que fue de $130,70 \mathrm{~g}$, siendo el de mayor peso el de categoría extra y el de menor, de la categoría corriente. Respecto a los ${ }^{-B}$ rix, se obtuvo como media un valor de 11,8 ${ }^{\circ}$ Brix y haciendo una comparación frente a las categorías, se registró el mismo comportamiento señalado para el peso promedio de fruto fresco. Los datos muestran una correspondencia respecto a las categorías, siendo mayor los ${ }^{\circ}$ Brix de la categoría extra, respecto a la categoría II (Tabla 2).

Tabla 2. Parámetros de calidad por categoría comercial para durazno Rubidoux en la Granja Experimental Tunguavita, Paipa, Boyacá

\begin{tabular}{lccc}
\hline CATEGORÍA & DIÁMETRO ECUATORIAL $(\mathbf{m m})$ & PESO FRESCO $(\mathbf{g})^{*}$ & ${ }^{\circ}$ Brix \\
\hline Extra & $>70$ & $186 \pm 13$ & $13,0 \pm 2,5$ \\
Primera (I) & $60-70$ & $145 \pm 17$ & $11,9 \pm 1,6$ \\
Segunda (II) & $50-70$ & $99 \pm 12$ & $11,1 \pm 1,9$ \\
Corriente & $<50$ & $69 \pm 12$ & - \\
\hline
\end{tabular}

*La variable consideró el peso promedio de frutos por categoría.

El peso fresco promedio por fruto y los ${ }^{\circ}$ Brix fueron registrados por pase (Tabla 3), para los dos parámetros en mención se refleja un mayor valor durante el pase 5. Los ${ }^{\circ}$ Brix aumentan progresivamente del pase uno al cinco en una proporción de 17\%, valor que declina en el último pase. De acuerdo al análisis de varianza no hay diferencias estadísticas para este parámetro entre pases. 
Tabla 3. Comportamiento de peso fresco y ${ }^{\circ}$ Brix de frutos de durazno Rubidoux por pase, en la Granja Experimental Tunguavita, Paipa, Boyacá

\begin{tabular}{lcccccc}
\hline Pase & $\mathbf{1}$ & $\mathbf{2}$ & $\mathbf{3}$ & $\mathbf{4}$ & $\mathbf{5}$ & $\mathbf{6}$ \\
\hline Peso $(\mathrm{g})^{*}$ & 141,28 & 127,89 & 114,72 & 129,69 & 148,32 & 122,80 \\
oBrix & $11,2 \pm 1,5$ & $11,3 \pm 2$ & $12,2 \pm 1,8$ & $12,4 \pm 2,5$ & $13,1 \pm 2,6$ & $12,1 \pm 2,2$ \\
\hline
\end{tabular}

*La variable consideró el peso promedio de fruto fresco por pase.

Respecto al contenido de BBix en relación con la coloración de la corteza, el análisis de varianza mostró diferencias estadísticas altamente significativas ( $p<0,001)$. Al realizar la comparación de medias se determinaron diferencias entre las tres categorías por color (Tabla 4). Adicionalmente se obtuvo una frecuencia relativa de $58,4 \%$ de frutos pintones, frente a un $36 \%$ y $5,8 \%$ de frutos pintónmaduro y maduro, respectivamente.

Tabla 4. ${ }^{\circ}$ Brix respecto al color de la piel de durazno Rubidoux en la Granja Experimental Tunguavita, Paipa, Boyacá

\begin{tabular}{lc}
\hline COLOR & ${ }^{\circ}$ BRIX* $^{*}$ \\
\hline Pintón & $10,6 \mathrm{a}$ \\
Pintón-Maduro & $11,7 \mathrm{~b}$ \\
Maduro & $13,6 \mathrm{c}$ \\
\hline
\end{tabular}

${ }^{*}$ Promedio con letras distintas indica diferencias significativas, según la prueba de Tukey $(p<0,05)$

\section{Discusión}

El color de la piel del durazno ha sido empleado junto con el conocimiento práctico de los agricultores, para determinar el momento de la cosecha de los frutos (Crisosto, 1994), así como se hizo en esta investigación, una vez que el agricultor poseedor de la cosecha fue quien determinó según parámetro visual y firmeza manual, la época de cosecha. La tabla de color realizada para este estudio fue útil para determinar por apreciación visual la madurez de los frutos evaluados. Normalmente el color de piel ha sido considerado como un parámetro no destructivo confiable para determinar el potencial de mercado del fruto, así como su vida útil, algunos autores han encontrado correlaciones altas entre el color y el grado de madurez de frutales caducifolios (Budde et al., 2003; Byrne et al., 1991; Shinya, Contador, Predieri, Rubio \& Infante, 2013)lu2018Kakamaslu2019 were harvested according to their visually assessed ground color and divided into four, ripeness classes (M1, M2, M3, and M4 correspondencia que se presentó de forma similar en esta investigación.

Es de notar que la observación y clasificación visual no son suficientes para determinar el grado de madurez, ya que la coloración superficial está determinada además del grado de madurez por la exposición a la luz solar (Lewallen \& Marini, 2003), por lo que otros autores han expresado que es preciso hacer este tipo de evaluaciones con ayuda de equipos electrónicos para determinar además del color de fondo medidas relacionadas con pigmentos que definen la madurez en durazno (Crisosto, 1994; Ferrer et al., 2005; Herrero-Langreo et al., 2012) y aún con mayor precisión emplear técnicas que permitan determinar el color de la pulpa ya que ésta se correlaciona en mayor medida con el grado de madurez del durazno (Crisosto et al., 2007). Aun así, la clasificación manual hecha a partir del color de la piel o corteza, de acuerdo a este estudio, se correspondió con la cantidad de -Brix presentes en los frutos. Estudios como el de Slaughter et al. (2006) demuestran que hay correspondencia hasta de un $83 \%$, tanto de técnicas manuales como instrumentales. para determinar parámetros de madurez de durazno y por lo tanto pueden ser consideradas adecuadas para tomar decisiones de cosecha, en campo. 
Respecto a parámetros de calidad de las diferentes variedades de duraznos en Colombia existe poca información, en especial para Rubidoux un estudio de la relación hoja-fruto refleja cómo el contenido de sólidos solubles totales y el peso fresco de los frutos está relacionado con la práctica de raleo de frutos. Para esta investigación se obtuvieron variaciones promedio a lo largo de la cosecha, en un rango aproximado entre $11^{\mathrm{a}}$ Brix y 13 Brix, se considera que las prácticas de raleo de fruto en la Granja Experimental Tunguavita son apropiadas, ya que se conoce que a mayor cantidad de hojas por fruto, es mayor el contenido de sólidos solubles en correspondencia con Casierra-posada et al. (2007) en donde se obtuvo un valor superior a 10Brix para frutos con más de 20 hojas.

De manera similar la cantidad de frutos obtenidos en la categoría primera, puede estar relacionada con la práctica de raleo de frutos, ya que el diámetro se aumenta con esta práctica (Casierra-posada et al., 2007), lo cual es determinante porque los consumidores tienen preferencia por productos de mayor tamaño y mayor contenido de azúcares. Así como se reporta para estudios de aceptación de durazno por los consumidores con base en el contenido de sólidos solubles, a partir de los cuales se registran preferencia mayores a $70 \%$ para valores superiores a $10^{\circ} \mathrm{Brix}$ en diferentes variedades de durazno (Crisosto \& Crisosto, 2005; Delgado, et al., 2013), es de recordar, que el contenido de azúcares registrado para Rubidoux es superior al mencionado, lo cual sugiere aceptación por los consumidores, aunque hay que precisar y hacer evaluaciones respecto a la acidez titulable. Cabe mencionar que la concentración de sólidos solubles igualmente está relacionada con el grado de exposición de los frutos a la luz (Lewallen \& Marini, 2003) lo que sugiere que prácticas de manejo como las podas, han sido implementadas adecuadamente en la Granja Tunguavita, permitiendo que los frutos tengan un cierto grado de uniformidad.

El comportamiento de peso fresco y ${ }^{\circ}$ Brix de frutos de durazno Rubidoux por pase, es esperado de acuerdo a otros reportes y a la fisiología de maduración del durazno (Ferrer et al., 2005). En cuatro variedades distintas de ciruela, se observó similar comportamiento para cinco y seis pases de cosecha con intervalos entre 6 y 8 días, con incrementos en los ${ }^{\circ}$ Brix entre un pase y otro (Usenik et al., 2008).

De otra parte, con referencia a los valores reportados para el pase cinco, se correspondieron con el período de mayor cantidad de frutos cosechados (datos no mostrados) lo que sugiere que fue el momento donde se obtuvo mayor homogeneidad comercial respecto al tamaño del fruto. Mientras en el último pase los valores decayeron, lo que puede estar relacionado con incidencia de lluvias, de acuerdo al IDEAM (2013) se tuvo un acumulado de $295,4 \mathrm{~mm}$ de lluvia durante la cosecha, de los cuales un $46 \%$ corresponden a los últimos 10 días. Para el brillo solar se presentó un comportamiento similar, el promedio de horas luz para el período evaluado fue 3,6 horas mientras para los últimos 10 días fue de 2,5. Estudios como el de Lewallen \& Marini (2003) constatan la dependencia entre el grado de exposición de los frutos a la luz y el contenido de azúcares. Resultados que adicionalmente, son coherentes con los de Alcobendas et al., (2013) quienes observaron diferencias estadísticas en oBrix y exposición a la luz, de manera que disminuyen a mayor cantidad de riego y menor exposición de los frutos de durazno a la luz solar. Finalmente Pinzón et al. (2014) señalan que los aspectos fisiológicos del duraznero están relacionados con cambios en las condiciones ambientales de la zona donde se producen.

\section{Conclusiones}

Los parámetros de calidad de durazno Rubidoux evaluados fueron consistentes con otros cultivares reportados en la literatura, de manera que las prácticas de precosecha y poscosecha implementadas en la Granja Experimental Tunguavita parecen estar acordes con los requerimientos del producto. Aun así, se precisa realizar a mayor 
profundidad la caracterización, incluyendo aspectos como firmeza del fruto y acidez titulable, que representen de manera consistente los índices de madurez para esta variedad, incluyendo el uso de técnicas no destructivas durante el proceso de maduración para poder determinar el momento oportuno de la cosecha.

Igualmente, teniendo en cuenta que el comportamiento entre cultivares varía, se sugiere llevar a cabo investigaciones que relacionen la madurez del producto con el grado de aceptación de los consumidores para su disposición final en el mercado, y también porque esto permitiría tomar decisiones de tipo agronómico que potencien algunas características deseables del fruto.

\section{Agradecimientos}

Las autoras expresan sus agradecimientos a la Dirección de Investigaciones DIN de la Universidad Pedagógica y Tecnológica de Colombia por el apoyo brindado al proyecto código SGI 1515, al personal de la Granja Experimental Tunguavita de la Uptc y al Centro de Innovación del Trópico Alto Sostenible-CEI3TAS.

\section{Literatura citada}

1. Alcobendas, R., Mirás-Avalos, J. M., Alarcón, J. J. \& Nicolás, E. (2013). Effects of irrigation and fruit position on size, colour, firmness and sugar contents of fruits in a mid-late maturing peach cultivar. Scientia Horticulturae, 164, 340-347.

2. Ávila, C., Robles, A., Pinzón, S., Miranda, D. \& Fisher, G. (2013). Tecnologías locales para los sistemas de producción de frutales caducifolios. En D. Miranda, G. Fischer, \& C. Carranza (Eds.), Los frutales caducifolios en Colombia: situación actual, sistemas de cultivo y planes de desarrollo (pp. 115-149). Bogotá, Colombia: Equilibrio Gráfico Editorial Ltda.

3. Budde, C. O., Tula, A., Polenta, G., Lucangeli, C., Murray, R., Iberoamericana, R., Murray, R. (2003). El color de fondo y la dureza como estimadores no destructivos de la firmeza de la pulpa en duraznos. Rev. Iber. Tecnología Postcosecha, 5(2), 134-139.

4. Byrne, D., Nikolic, A. \& Burns, E. (1991). Variability in sugars, acids, firmness, and color characteristics of 12 peach genotypes. Journal of the American Society for Horticultural Science, 116(6), 1004-1006.

5. Casierra-Posada, F., Rodríguez-Puerto, J. \& CárdenasHernández, J. (2007). La relación hoja:fruto afecta la producción, el crecimiento y la calidad del fruto en duraznero (Prunus persica L. Batsch cv. "Rubidoux"). Rev. Fac.Nal.Agr.Medellín, 60(1), 3657-3669.

6. Casierra-Posada, F. \& Aguilar-Avendaño, O. (2008). Calidad en frutos de tomate (Solanum lycopersicum L.) cosechados en diferentes estados de madurez. Agronomía Colombiana, 26(2), 300-307.

7. Crisosto, C., Lurie, S. \& Retamales, J. (2009). Stone fruits, En: E. Yahia (Eds), Modified and controlled atmospheres for the storage, transportation and packaging of horticultural commodities (pp. 287-315). Boca Raton, Florida: Taylor \&Francis group, LLC.

8. Crisosto, C. \& Costa, G. (2008). Preharvest Factors Affecting Peach Quality. En D. Layne \& D. Bassie (Eds.), The peach: botany, production and uses (pp. 536-549). UK: CAB

9. Crisosto, C. (1994). Stone fruit maturity indices : a descriptive review. Postharvest News and Information, 5(6), 65-69.

10. Crisosto, C. H. \& Crisosto, G. M. (2005). Relationship between ripe soluble solids concentration (RSSC) and consumer acceptance of high and low acid melting flesh peach and nectarine (Prunus persica (L.) Batsch) cultivars. Postharvest Biology and Technology, 38(3), 239-246.

11. Crisosto, C. H., Valero, C. \& Slaughter, D. C. (2007). Predicting pitting damage during processing in californian clingstone peaches using color and firmness measurements. Applied Engineering in Agriculture, 23(2), 1-6.

12. Crisosto, C. \& Valero, D. (2008). Harvesting and postharvest handling of dates. En D. Layne \& D. Bassie (Eds.), The peach: botany, production and uses (pp. 576-596). UK: CAB International.

13. Dagar, A., Weksler, A., Friedman, H., Ogundiwin, E. A., Crisosto, C. H., Ahmad, R. \& Lurie, S. (2011). Comparing ripening and storage characteristics of "Oded" peach and its nectarine mutant "Yuval." Postharvest Biology and Technology, 60(1), 1-6.

14. 14) Daza, A., García-Galavís, P. A., Grande, M. J. \& Santamaría, C. (2008). Fruit quality parameters of "Pioneer" Japanese plums produced on eight different rootstocks. Scientia Horticulturae, 118(3), 206-211.

15. Delgado, C., Crisosto, G. M., Heymann, H. \& Crisosto, C. H. (2013). Determining the Primary Drivers of Liking to Predict Consumers' Acceptance of Fresh Nectarines and Peaches. Journal of Food Science, 78(4), S605-S614.

16. Ferrer, A., Remón, S., Negueruela, A. I. \& Oria, R. (2005). Changes during the ripening of the very late season Spanish peach cultivar Calanda: Feasibility of using CIELAB coordinates as maturity indices. Scientia Horticulturae, 105(4), 435-446.

17. Herrero-Langreo, A., Fernández-Ahumada, E., Roger, J.-M., Palagós, B. \& Lleó, L. (2012). Combination of optical and non-destructive mechanical techniques for the measurement of maturity in peach. Journal of Food Engineering, 108(1), 150-157. 
18. Instituto de Hidrología, Meteorología y Estudios Ambientales- IDEAM. (2013). Información hidrometeorológica, Base de datos. Bogotá, Colombia.

19. Jha, S. N., Narsaiah, K., Sharma, A. D., Singh, M., Bansal, S. \& Kumar, R. (2010). Quality parameters of mango and potential of non-destructive techniques for their measurement - A review. Journal of Food Science and Technology, 47(1), 1-14.

20. Kays, S. J. (1999). Preharvest factors affecting appearance. Postharvest Biology and Technology, 15(3), 233-247.

21. Lewallen, K. S. \& Marini, R. P. (2003). Relationship between Flesh Firmness and Ground Color in Peach as Influenced by Light and Canopy Position. Journal of American Society Horticultural Science, 128(July 1999), 163-170.

22. Ministerio de Agricultura y Desarrollo Rural-MADR. (2012). Anuario estadístico de frutas y hortalizas 20072011. Recuperado de http://207.239.251.112/www/htm3b/ public/Anuario/ANUARIO\%20ESTADISTICO\%20 DE\%20FRUTAS\%20Y\%20HORTALIZAS\%202011.pdf

23. Miranda D. \& Carranza, C. (2013) Caracterización, clasificacion y tipificación de sistemas productivos de caducifolios, con énfasis en duraznero, manzano, ciruelo y peral, En: D. Miranda, G. Fischer \& C. Carranza (Eds), Los frutales caducifolios en Colombia: situación actual, sistemas de cultivo y planes de desarrollo (pp. 87-113). Bogotá, Colombia: Equilibrio Gráfico Editorial Ltda.

24. Pérez-Marín, D., Sánchez, M. T., Paz, P., Soriano, M. A., Guerrero, J. E. \& Garrido-Varo, A. (2009). Non-destructive determination of quality parameters in nectarines during on-tree ripening and postharvest storage. Postharvest Biology and Technology, 52(2), 180-188.

25. Pinzón, E., Morillo, A. \& Fischer, G. (2014). Aspectos fisiológicos del duraznero ( Prunus persica [ $\mathrm{L}$.] Batsch ) en el trópico alto: una revisión. Revista U.D.C.A. Actualidad \& Divulgación Científica, 17(2), 401-411.
26. Puentes, G. A., Felipe, L. \& Teresa, L. (2008). Análisis de grupo de las empresas productoras de frutales caducifolios del departamento de Boyacá. Agronomía Colombiana, 26(1), 146-154.

27. Reid, M. (2002). Maturation and maturity indices. En: A. Kader (Ed), Postharvest technology of horticultural crops (pp. 55-62). University of California, Oakland, CA, USA: ANR

28. Shinya, P., Contador, L., Predieri, S., Rubio, P. \& Infante, R. (2013). Peach ripening: Segregation at harvest and postharvest flesh softening. Postharvest Biology and Technology, 86, 472-478.

29. Slaughter, D. C., Crisosto, C. H., Hasey, J. K. \& Thompson, J. F. (2006). Comparison of instrumental and manual inspection of clingstone peaches. Applied Engineering in Agriculture, 22(6), 883-889.

30. Tijskens, L. M. M., Zerbini, P. E., Schouten, R. E., Vanoli, M., Jacob, S., Grassi, M. \& Torricelli, A. (2007). Assessing harvest maturity in nectarines. Postharvest Biology and Technology, 45(2), 204-213.

31. Usenik, V., Kastelec, D., Veberi凶, R. \& Štampar, F. (2008). Quality changes during ripening of plums (Prunus domestica L.). Food Chemistry, 111(4), 830-836.

32. Weber M., Güemes D., Pirovani M., Piagentini A., Zanuttini A. \& Gariglio N. (2003). Características del fruto del duraznero 'flordaking' cultivado en la zona centroeste de la provincia de Santa Fe (Argentina). Revista FAVE-Ciencias Agrarias, 2, 29-35.

\section{Conflicto de Intereses}

Los autores declaran no tener ningún conflicto de intereses 
